\title{
A Laparoscopic Assisted Minilaparotomy Hysterectomy approach for Very Large Fibroid Uteri of more than 1000 grams - A Preliminary Study Wong WSF
}

School of Women and Child Health, University of New South Wales, Sydney, Australia

${ }^{*}$ Corresponding author: Wong WSF, School of Women and Child Health, University of New South Wales, Sydney, Australia, E-mail: Fwong3@hotmail.com.hk

Citation: Wong WSF (2015) A Laparoscopic Assisted Minilaparotomy Hysterectomy approach for Very Large Fibroid Uteri of more than 1000 grams - A Preliminary Study. J Gynecol Res 1(1): 101. doi: 10.15744/24543284.1.101

Received Date: February 04, 2015 Accepted Date: April 20, 2015 Published Date: April 24, 2015

\begin{abstract}
Objective: To report the surgical outcome of a laparoscopic assisted minilaparotomy hysterectomy (LMH) surgical approach for the management of very large fibroid uteri.

Setting: A private hospital, St. Paul's Hospital, in Hong Kong

Patients and Surgical techniques: It is a retrospective study to assess a hysterectomy approach for very large fibroid uteri of more than 1000 grams. This paper described the surgical technique of this hysterectomy approach and presented the patient characteristics, surgical data and clinical outcome.

Result: From 2011 to 2014, 13 women underwent a surgical approach of laparoscopic assisted minilaparotomy hysterectomy with or without salpingo-oophorectomy (LMH +/- SO) for very large fibroid uteri larger than 1000 grams. All hysterectomies were successfully completed without intraoperative or postoperative complication. The amount of blood loss was comparatively less than that of abdominal hysterectomy for very large fibroid uteri reported in the literature. All patients recovered well after operation and many had an early discharge from hospital.

Conclusion: In conclusion, this surgical approach for hysterectomy can replace open abdominal hysterectomy for very large fibroid uteri more than 1000 grams. The technique is a combination of laparoscopy and minilaparotomy hysterectomy. It is a safe and feasible alternative as a minimally invasive surgery for very large fibroid uteri.
\end{abstract}

Keywords: Laparoscopic assisted; Minilaparotomy; Hysterectomy; Large uterus > 1000 grams

\section{Introduction}

Minilaparotomy has been used for tubal sterilization for decades. It became a new surgical idea described by some who performed minilaparotomy hysterectomy and achieved outcomes that were less invasive than those of traditional open laparotomy [1-3]. Pelosi and Pelosi 2004 reported a minilaparotomy procedure in patients in whom vaginal hysterectomy was considered contraindicated [4]. Their procedure offered a safe alternative with minimal access and good outcome. Its principle of uses relied on traditional open techniques with a small incision and laparotomy instrumentation, and avoided the use of a large abdominal open wound or expensive laparoscopic equipment. Pelosi 2004 claimed that there was no long learning curve and that the procedure resulted in reduced operating time and a shorter recovery time similar to that of minimally invasive surgery [4]. Since then, minilaparotomy hysterectomy has been reported by many authors as a safe and feasible route of hysterectomy [5-9]. However, this minilaparotomy approach might still be considered difficult for the very large fibroid uteri because of the difficulty to assess the sizes and locations of fibroids, presence of adhesions and the iceberg phenomenon of the large fibroid uterus within the pelvis.

On the other hands, some laparoscopists would also find hysterectomy for very large fibroid uteri difficult to perform. The laparoscopic hysterectomy for large fibroid is known to be difficult surgeries with long operating time, increased blood loss sometimes requiring blood transfusion or conversions to open laparotomy [10-12].

In this paper, the author reported his personal small series of performing 13 hysterectomies for very large fibroid uteri of more than 1000 grams using a combined laparoscopy and minilaparotomy approach. In his experience, he found that this combined approach was feasible, safe and practical without a long learning curve.

\section{Patients and surgical techniques}

From 2011 to 2014, 13 patients with very large fibroid uteri of greater than 1000 grams underwent hysterectomy with a laparoscopic assisted minilaparotomy approach. Their characteristics and surgical outcome were presented in Table 1.

The surgical techniques were described in detail as follow 
Direct portal entry technique: The narrow peritoneal space between a large fibroid uterus and the abdominal wall made it difficult for the insertion of a Veress needle to create a penumo-peritoneum for the insertion of a trocar and cannula. Although Hassan's open technique could be used, the approach used in this paper was a direct entry technique under the laparoscopic vision. No prior $\mathrm{CO}_{2}$ insufflation with a Veress needle was required. A $5 \mathrm{~mm}$ Endopath Xcel trocar device was introduced through the umbilicus under direct laparoscopic vision with real time image monitoring. After the rectus sheath and the peritoneum were seen penetrating through, the trocar could be seen positioned in the narrow space between the abdominal wall and the large uterus. The Xcel trocar with the laparoscope was then withdrawn and $\mathrm{CO}_{2}$ gas insufflation started. The laparoscope was then reintroduced through the Xcel cannula for the inspection of the abdominal and pelvic organs. The direct entry techniques had been reported previously [13]. All direct entries in this series were successful and uneventful. Apart from the use of a $5 \mathrm{~mm}$ laparoscope (both 0 degree and 30 degree laparoscopes could be used depending on their availability), the use of $\mathrm{CO}_{2}$ insufflation was brief and lasted till direct laparoscopic inspection was completed prior to the mini-laparotomy. Once the mini-laparotomy wound was made, no more gas insufflation was required.

Minilaparotomy hysterectomy: The patient was positioned in a Trendelenburg lithotomy position. The minilaparotomy technique consisted of making a 4-6 cm suprapubic incision at the level of or just below the pubic hair line, the incision of the subcutaneous fat to the rectus sheath which was then cut opened transversely. The underlying rectus muscles were then pulled apart to expose the underlying peritoneum. The peritoneum was incised sharply and stretched opened. A self-retaining Alexis wound protector/ retractor of size 2.5-6 cm (Applied Medical, Rancho Santa Margarita, CA, USA), was inserted into the wound to provide traction and protection. The elastic traction force of the protector aided in further opening of the abdominal wound (Figure 1a).

Maximal exposure of the surgical field could be achieved by positioning one of two small Heaney or Deaver retractors to the wound protector to enlarge or lift up the wound in any one direction. Lateral sideway displacements of the uterus could also be manipulated by using a uterine manipulator. If adhesions were encountered, open surgical technique and instruments were used to lyse the adhesions. Using this minilaparotomy approach, the round ligaments, tubes or ovarian infundibulo-pelvic ligaments could be coagulated and divided under vision when they were now easily visualized and accessible. Vessels sealing devices like LigaSure (Covidien AG), Erbe BiClamp (ERBE Elektromedizin GmbH) or other laparoscopic bipolar forceps could be used as in laparoscopy but via the lifted up mini-laparotomy wound. In this way, coagulation of ovarian or uterine vessels and occasional large superficial vessel over the fibroid could be done without the use of laparoscopic operating ports.

For very large fibroid uteri, tissue reduction might be necessary through the minilaparotomy wound. Then myomectomy was performed to remove some fibroids so as to reduce the size of the uterus. After the bladder peritoneum was incised and the bladder stripped downward as in abdominal surgery, another technique used was to identify the two uterine arteries on both sides of the cervix, coagulated them or put a suture around them prior to perform a subtotal hysterectomy above that level of the upper cervix. The large fibroid uterus was then removed with a 'paper roll' morcellation technique (Figure 1b) as previously reported by the author [14]. Briefly the 'paper roll' technique was performed as follow - two small Heaney retractors were placed at 6 o'clock and 10 o'clock positions of Alexis wound retractor. Two vulsellum forceps were used to grasp the fibroid uterus which had been separated from the cervix after the subtotal hysterectomy procedure. A No. 10 sharp scalpel was used to cut into the fibroid uterus, with a continued upward traction as much uterine tissue as possible was removed out of the minilaparotomy wound during the cut. The incision should be in a direction beginning from the Heaney retractor at the 10 oclock and ending at the other retractor at the 6 o'clock. A 2.0 to $2.5 \mathrm{~cm}$ of tubular incised uterine tissue was delivered under traction at the time of the cut, the remaining portion of the uterus within the abdomen should simultaneously be rotated to allow more uterine tissues to roll and cut like a paper roll being pulled out. Care is taken not to cut or damage the Alexis wound retractor, thus losing pieces of cut plastics unintentionally into the abdominal cavity. All cuts were made using a sharp cold knife under direct vision at the level of the abdominal wound. Pulling traction was maintained at all times during the incision. This manoeuver could remove a very large fibroid uterus without difficulty in a relatively short time of 10-15 minutes. It is also possible to remove the entire large uterus (without the cervix) in 1 piece regardless of its size. The remaining cervix was last removed after the complete delivery of the large fibroid uterus through the minilaparotomy wound. Figure $1 \mathrm{c}$ and $1 \mathrm{~d}$ showed the abdominal appearance before and after the hysterectomy with this combined laparotomy and minilaparotomy approach.

\section{Result}

Table 1 showed the patients' characteristics, uterine size and weight, operation data, complications and length of hospital stay in this series of patients. The average age of patients was 49+/- 6.3 (32-52 years) and their median BMI was 23 +/- 4.4 (17.30-29.38). The median size and weight of the uteri was 20 gravid week size (range 16 to 30 weeks) and $1100+/-511.2$ grams (1010 to 2900 grams). The median duration of operations, blood loss and length of hospital stay were $2+/-0.4$ hours (1.5 to 3 hours), $300+/-206$ $\mathrm{ml}(150$ to $800 \mathrm{ml})$ and $2.0+/-1.0$ days (1.5 to 4 days) respectively.

There was no fever, postoperative antibiotic uses or any injuries in these patients. A patient received three units of blood transfusion prior to her operation because of her pre-operative haemoglobin level was $6.3 \mathrm{~g} \%$. No patient required blood transfusion due to blood loss at operation. All patients could be mobilized on day 1 after her operation with the use of oral analgesic and 11 out of 13 were discharged home within 3 days after their operations. 


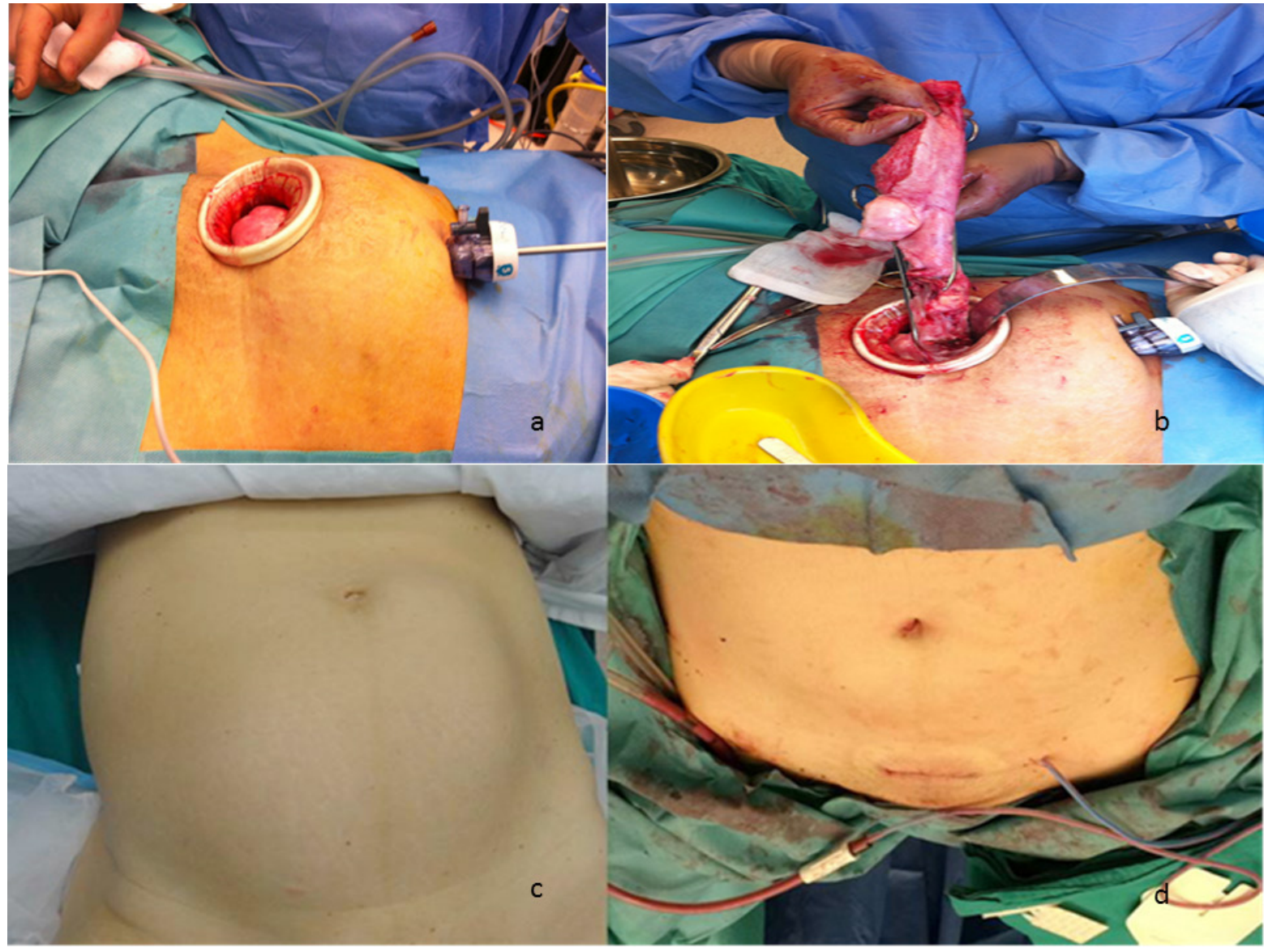

Figure 1a: A self-retaining Alexis $\mathrm{R}$ wound protector and laparoscope in place for the procedure; 1b) A 'paper roll' morcellation technique to remove a very large fibroid through the minilaparotomy wound; 1c) and 1d) The abdominal appearances before and after the removal of a very large fibroid uterus with a combined laparoscopy and minilaparotomy hysterectomy approach.

\begin{tabular}{|c|c|c|c|c|c|c|c|c|c|}
\hline & Age & BMI & $\begin{array}{l}\text { Uterine size } \\
\text { (weeks) }\end{array}$ & $\begin{array}{l}\text { Uterine weight } \\
\text { (grams) }\end{array}$ & Type of Surgery & Complications & Length of stay & $\begin{array}{l}\text { OT time } \\
\text { (hrs) }\end{array}$ & $\begin{array}{c}\text { Blood loss } \\
(\mathrm{ml})\end{array}$ \\
\hline 1 & 50 & 23.02 & 18 & 1010 & LMHBSO & 0 & 3 & 2 & 150 \\
\hline 2 & 46 & 25.04 & 16 & 1050 & LMH & 0 & 1.5 & 2 & 500 \\
\hline 3 & 52 & 22.60 & 30 & 2900 & LMHBSO & 0 & 2 & 3 & 500 \\
\hline 4 & 51 & 21.16 & 18 & 1080 & LMHBSO & 0 & 2 & 1.5 & 200 \\
\hline 5 & 48 & 29.17 & 24 & 1530 & LMHBSO & 0 & 3 & 2 & 200 \\
\hline 6 & 49 & 17.85 & 28 & 1605 & LMHBSO & 0 & 2 & 2.5 & 500 \\
\hline 7 & 32 & 17.30 & 20 & 1100 & LMH & 0 & 3 & 1.5 & 800 \\
\hline 8 & 52 & 27.29 & 18 & 1230 & LMHBSO & 0 & 3 & 1.5 & 500 \\
\hline 9 & 35 & 29.38 & 18 & 1020 & LMH & 0 & 4 & 2 & 200 \\
\hline 10 & 42 & 17.94 & 20 & 1050 & LMH & 0 & 2 & 1.5 & 300 \\
\hline 11 & 49 & 23.57 & 20 & 1279 & LMHBSO & 0 & 5 & 2 & 200 \\
\hline 12 & 48 & 29.34 & 18 & 1070 & LMHROC & 0 & 1.5 & 1.5 & 100 \\
\hline 13 & 50 & 19.35 & 22 & 1250 & LMHBSO & 0 & 2 & 2.5 & 500 \\
\hline Median & 49 & 23.02 & 20 & 1100 & & & 2 & 2 & 300 \\
\hline $\begin{array}{l}\text { Standard } \\
\text { Deviation }\end{array}$ & 6.36 & 4.49 & 4.21 & 511.27 & & & 1.02 & 0.48 & 206.00 \\
\hline
\end{tabular}

LMH - Laparoscopic assisted minilaparotomy hysterectomy

BSO - Bilateral salpingo-oophorectomy

ROC - Right ovarian cystectomy

Table 1: Patients' characteristics and surgical outcomes 


\section{Discussion}

Hillis et al 1996 found a 60\% complication rate of abdominal hysterectomy in patients with fibroid uteri over 500 grams [15]. This was also confirmed by Unger et al 2002 that there was a significant increase in perioperative complications for women with large uterus. They reported an overall complication rate of $61.7 \%$ for uteri over 1000 grams and $41.3 \%$ in the group over 500 grams [16]. The complication rate from abdominal hysterectomy increased due mainly to an increased blood loss associated with surgery. Unger et al 2002 found 55\% (26 of 47) women undergoing abdominal hysterectomy with uterine weight greater than 1,000 grams had estimated blood loss greater than $500 \mathrm{ml}$.

Very large fibroid uteri are usually removed via large vertical incisions and have conventionally been considered to be a relative contraindication for vaginal and laparoscopic hysterectomy. However several publications had reported on the feasibility of the laparoscopic approach for removal of fibroid uteri weighing more than 500 grams [14,17]. However, this laparoscopic surgical procedure is for the very skilled laparoscopic surgeons. Therefore, the laparoscopic approach has not become the standard of care for these challenging cases with large fibroid uteri, possibly because advanced laparoscopic skills and the long training period to acquire these skills. Thus, it seems reasonable to explore other minimally invasive techniques that could potentially be employed to treat patients with very large fibroid uteri by general gynecologists.

The combined use of laparoscope and minilaparotomy is not a new concept. In 1995, Wood and Maher reported a technique of laparoscopic minilaparotomy hysterectomy using a "Maher" abdominal elevator to elevate the abdomen to perform the hysterectomy. This gasless laparoscopy approach used a $2 \mathrm{~cm}$ incision in the lower abdomen for the abdominal procedure. They used the laparoscopic visualization of the pelvis to guide their fingers and instruments through the $2 \mathrm{~cm}$ minilaparotomy wound to enucleate myomas, to free any adherent ovaries, to identify and dissect endometriotic nodules in the Pouch of Douglas. They reported three patients with recurrent endometriosis in AFS stage 3 and 4 having this combined procedure performed. The uterus was manipulated by a curette inserted vaginally into the uterine cavity. Finger and scissors introduced through the minilaparotomy were used in dissection, to identify the anatomy and pathology as practiced in laparotomy. In this approach, they used open laparotomy techniques and instruments to ligate and suture pedicles. They reported no postoperative complications, reduced postoperative pain, smaller incision and early discharge from hospital as compared to laparotomy [18].

Koh et al 2008 also used a combination of approaches and reported the removal of a very large 3250 grams myomatous uterus by minilaparotomy and laparoscopic assisted vaginal hysterectomy (LAVH) [19]. They placed the first trocar entry at the Palmer's point. The accessary operating ports were positioned laterally on both sides of the umbilicus. The laparoscopic part involved the coagulation and division of both round ligaments and other uterine suspensory ligaments. A vaginal approach was then taken to separate the uterus from the supporting cardinal and uterosacral ligaments. Through a $7 \mathrm{~cm}$ transverse suprapubic minilaparotomy wound, the freed uterus was morcellated by a scalpel and removed. They then closed the vaginal vault transvaginally and the minilaparotomy wound abdominally to complete the procedure.

The advantages of the present approach are also the uses of a combination of open abdominal surgical techniques and laparoscopic vessel sealing device or electrosurgical instruments. These new instrumentations facilitate this minilaparotomy approach to improve the hemostasis and reduce the operation time. As a result, the blood loss reported in this series for the very large fibroids were comparably lower than the open abdominal procedures and equivalent to the laparoscopic procedures reported in the literature $[14,16,20]$. Therefore instead of using suturing and cut approach as in open abdominal surgery with a large wound, the use of these vessel sealing devices can coagulate and divide vessels and tubes from a distance using the mini-laparotomy wound under a direct vision. Even without much training, surgeons with proficient abdominal surgical skill can operate through a minilaparotomy wound under laparoscopic guided vision. The duration of surgery, volume of blood loss and length of hospital stay were demonstrated to be acceptable as in this series.

Many including the author had previously reported a comparable outcome between the minilaparotomy hysterectomy and the laparoscopic hysterectomy. Even with the traditional minilaparotomy hysterectomy approach, it could be difficult to perform a hysterectomy for a very large fibroid uterus. It is because through a small minilaparotomy wound the surgery could still be hard to perform without adequate space. Except for those very few skilled surgeons, many would rather enlarge the wound to perform an abdominal hysterectomy with a larger wound. The adoption of this combined laparoscopy and minilaparotomy approach, the difficulties of minilaparotomy for very large fibroid uteri could be resolved.

The use of a direct entry technique under laparoscopic vision makes it easier to enter into the narrow space between the enlarged uterus and the abdominal wall. Thus it can result in a hidden umbilical wound without an obvious portal wound as in other alternative abdominal portal entry like Lee-Huang point entry (mid-point between Xiphoid sternum and umbilicus) or left upper quadrant entry.

Nevertheless this combined laparotomy and minilaparotomy approach should not be used for hysterectomy that can be easily performed by other approach. Minilaparotomy alone using a small wound is by itself still a laparotomy, and it has its complications. In general, the laparoscopic approach should be preferred to minilaparotomy hysterectomy when the vaginal approach cannot be used [21]. This combined approach only plays a role in situation when laparoscopy is seemed to be beyond the skill of the surgeons such as in cases of very large fibroid uteri. Unlike the report from Koh and Koh [19], this present approach did not use additional 
ports to perform the operation. As a result, the only visible scars were the small umbilical scar and the minilaparotomy scar hidden below the pelvic hairline. Many surgeons who perform a total laparoscopic hysterectomy for very large fibroid, may still make a suprapubic wound to remove the large fibroid uterus in order to save time and reduce bleeding. Although the author did report successful LAVH procedure for fibroids $>500$ grams with a largest uterus $>1600$ grams successfully [14], the LAVH technique for very large fibroid uterus was still difficult compared to this combined approach.

Today, with proper pre-operative ultrasound assessment, the size of the fibroids or uterus can be easily determined. As a simple rule, when the fundus of the uterus is palpable at or above the level of the umbilicus, the uterus or large fibroids can easily be close to 1000 grams or more. A combined minilaparotomy and laparoscopy approach can be then chosen when the routine laparoscope and vaginal hysterectomy are found to be difficult or not feasible depending on the surgeon's surgical competency. For a gynaecologist who is already experienced in open surgery, he/she only needs slight adjustment and modification of the open abdominal technique and standard laparoscopy skill to become proficient in this combined laparoscopy and minilaparotomy approach. Therefore this approach may be more suitable to remove very large uteri for many gynaecologists because it does not require specialized endoscopic skill and advanced endoscopic training.

This preliminary report is limited by its small number, retrospective and nonrandomized design. This is because it is a single surgeon's private practice and his preference to use this approach for very large uteri. As all the operations were performed by him, the present results may not be reproducible in other general gynaecological practice. The small number of recruited patients is also certainly underpowered to look at major complications of this hysterectomy approach. Future prospective, multicentres and randomized studies are needed to assess whether this laparoscopic assisted minilaparotomy hysterectomy approach is associated with low complications, ease to perform and acceptability by patients.

Finally, here are some words of warning about the management of these very large fibroid uteri. Patients with giant fibroid uteri present an unusual challenge. Even though many of these patients are relatively young, they might be severely anemic or malnourished. They require proper perioperative assessment and management to prevent morbidity and to ensure a speedy recovery. It should also be noted that suspicious large fibroids must be treated with prudence because of the rare possibility of a leiomyosarcoma. The latter might present with continuing growth in menopausal women, on GnRH agonist treatment, rapid growth over a short time frame, and appearing suspicious on ultrasound or MRI scans. This approach would help to minimize the risk of electrical morcellation in the dissemination of malignancy which has been so widely debated in recent time[22].

\section{Conclusion}

Although the present study is limited by its retrospective and nonrandomized design, but in conclusion, it had demonstrated the feasibility, safety and practicality of a combined laparoscopy and minilaparotomy hysterectomy approach for large uteri of 1000 grams or more.

\section{References}

1. Benedetti-Panici P, Maneschi F, Cutillo G, Scambia G, Congiu M, et al. (1996) Surgery by minilaparotomy in benign gynecologic disease. Obstet Gynecol 87: 456-9.

2. Chalkoo M, Ahangar S, Durrani AM, Shah MJ, Habib R, et al. (2011) Mini-lap hysterectomy revisited with new concepts and technical modifications. Int J Surg 9: 404-9.

3. Panici PB, Zullo MA, Angioli R, Muzii L (2005) Minilaparotomy hysterectomy: a valid option for the treatment of benign uterine pathologies. Eur J Obstet Gynecol Reprod Biol 119: 228-31.

4. Pelosi $2^{\text {nd }} M$, Pelosi $3^{\text {rd }} M(2004)$ Pelosi minilaparotomy hysterectomy: a non-endoscopic minimally invasive alternative to laparoscopy and laparotomy. Surg Technol Int 13: 157-67.

5. Wong WSF, Lee TCE (2011) A Retrospective Series of Laparoscopic Hysterectomy (LH) and Modified Mini-laparotomy Abdominal Hysterectomy (MAH) for the Management of Enlarged Uterus - a Reappraisal of the Surgical Approach for Hysterectomy. Chin J Minim Invasive Surg 11: 289-94

6. Hoffman M, Lynch C (1998) Minilaparotomy hysterectomy. Am J Obs Gynecol 179: 316-20.

7. Li-xia P, Ming-huo Y (2006) Clinical evaluation of mini laparotomy intrafascial hysterectomy. Chongqing Medicine 11.

8. Royo P, Alcázar JL, García-Manero M, Olartecoechea B, López-García G (2009) The value of minilaparotomy for total hysterectomy for benign uterine disease: a comparative study with conventional Pfannenstiel and laparoscopic approaches. Int Arch Med 2: 11.

9. Sharma JB, Wadhwa L, Malhotra M, Arora R (2004) Mini laparotomy versus conventional laparotomy for abdominal hysterectomy: A comparative study. Indian J Med Sci 58: 196-202.

10. Lyons TL, Adolph AJ, Winer WK (2004) Laparoscopic supracervical hysterectomy for the large uterus. J Am Assoc Gynecol Laparosc 11: 170-4.

11. O'Shea RT, Cook JR, Seman EI (2002) Total laparoscopic hysterectomy: a new option for removal of the large myomatous uterus. Aust N Z J Obstet Gynaecol 42: $282-4$.

12. Pelosi MA, Kadar N (1994) Laparoscopically assisted hysterectomy for uteri weighing $500 \mathrm{~g}$ or more. J Am Assoc Gynecol Laparosc 1: 405-9.

13. Wong WSF (2013) A safe optically guided entry technique using Endopath Xcel Trocars in laparoscopic surgery: A personal series of 821 patients. Gynecol Minim Invasive The 2: 30-3.

14. Wong WSF, Lee TCE, Lim CED (2010) Novel vaginal "paper roll" uterine morcellation technique for removal of large (> 500 g) uterus. J minimal invasive gynecol 17: 374-8. 
15. Hillis SD, Marchbanks PA, Peterson HB (1996) Uterine size and risk of complications among women undergoing abdominal hysterectomy for leiomyomas. Obstet Gynecol 87: 539-43.

16. Unger JB, Paul R, Caldito G (2002) Hysterectomy for the massive leiomyomatous uterus. Obstet Gynecol 100: $1271-5$.

17. Walid MS, Heaton RL (2010) Total Laparoscopic Hysterectomy for Uteri Over One Kilogram. JSLS 14: 178-82.

18. Wood C, Maher P (1995) Laparoscopic Minilaparotomy Hysterectomy. Aus N Z J Obstet Gynaecol 35: 204-7.

19. Koh LW, Koh PR, Wong CN, Sun YL, Chang TP, et al. (2008) Minilaparotomy-Assisted LAVH for a Very Large Fibroid. JSLS 12: 417-9.

20. Shiota M, Kotani Y, Umemoto M, Tobiume T, Shimaoka M, et al. (2011) Total abdominal hysterectomy versus laparoscopically-assisted vaginal hysterectomy versus total vaginal hysterectomy. Asian J Endosc Surg 4: 161-5.

21. Muzii L, Basile S, Zupi E, Marconi D, Zullo MA, et al. (2007) Laparoscopic-assisted vaginal hysterectomy versus minilaparotomy hysterectomy: a prospective, randomized, multicenter study. J Minim Invasive Gynecol 14: 610-5.

22. Kho KA, Nezhat CH (2014) Evaluating the risks of electric uterine morcellation. JAMA 311: 905-6.

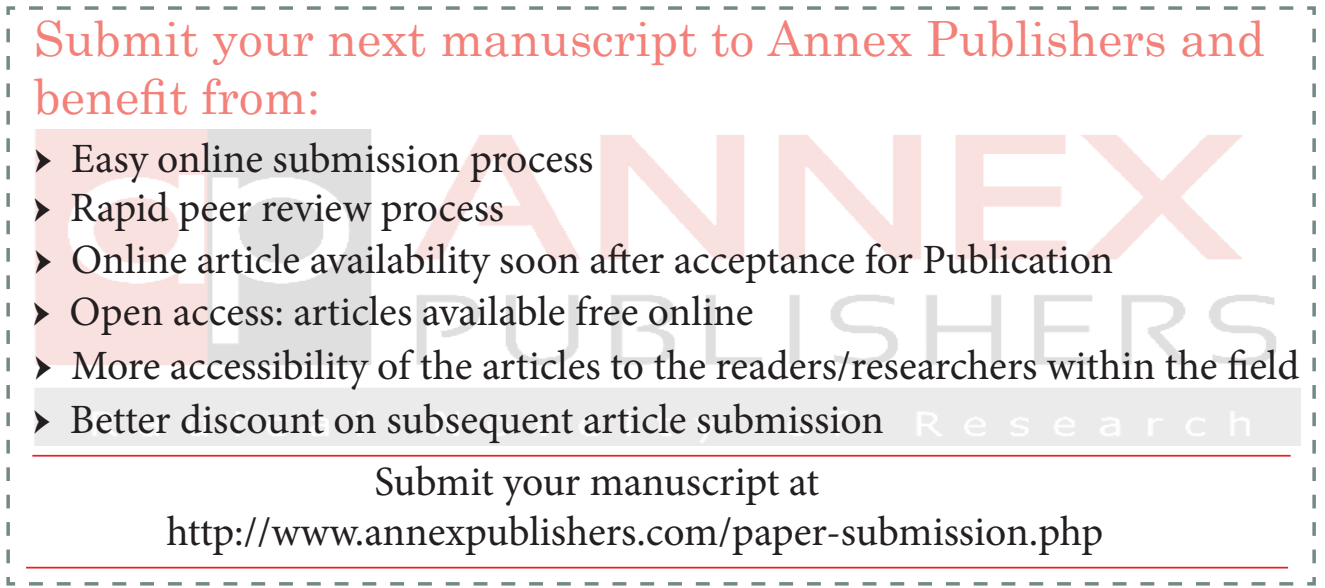

